\title{
The visualization of fluid content during intestinal MRI
}

\author{
Olga A.Subbotina ${ }^{1}$, Andrey Yu. Letyagin ${ }^{1,2}$, Mariya V. Rezakova ${ }^{1}$ \\ ${ }^{1}$ Department of MRI, State Scientific-Research Institute of Physiology and Basic Medicine \\ Novosibirsk, Russia \\ o.a.subbotina@yandex.ru \\ ${ }^{2}$ Research Institute of Clinical and Experimental Lymphology - Branch of the Institute of Cytology and Genetics SB RAS \\ Novosibirsk, Russia \\ letygin-andrey@yandex.ru
}

\begin{abstract}
MRI, detailed visualization of the intestine is not possible. Using the MRI technique with peroral hydrocontrast, it becomes possible to analyze the state of the intestine along its entire length due to its liquid content, biphasic contrast and obtaining a balanced MR signal from the intestinal wall with a high $\mathrm{T} 1$ / $\mathrm{T} 2$ ratio.
\end{abstract}

Keywords - intestinal MRI, Hydro-MRI, MR-enterography, peroral contrast

\section{INTRODUCTION}

In 2016, clinical recommendations were issued of the Russian Gastroenterological Association and the Association of Russian Coloproctologists for the diagnosis and treatment of Crohn's disease, where MR enterography is one of the methods for diagnosing and monitoring the effectiveness of treatment, as well as with suspected presence of stenosis and fistulas. Of course, the diagnostic capabilities of hydro-MRI are much wider and capture a greater number of nosologies, including not only other types of inflammatory bowel diseases, but also a number of infectious diarrhea, tumor pathology. Since the advent of MRI, visualization of the gastrointestinal tract was considered beyond the boundaries of this method, but hydrocontrasting of the intestinal lumen with special peroral contrasts made it possible to visualize and analyze it. The determination of fluid content in MRI required the use of modified signal sequences, which became the basis of the new method.

\section{MATERIALS AND METHODS}

When receiving hydrocontrast, different types of peroral contrasts are used: negative, positive and biphasic. Each of them has its own advantages and disadvantages. Negative contrasts have a hypo-intense signal both on the T1-weighted image (T1-WI) and on the T2-weighted image (T2-WI), so the intestinal lumen is always dark. This is an indisputable advantage in assessing the intestinal wall with contrast enhancement and the contrasting pattern (white on dark), but leads to an inadequate assessment of the thickness of the intestinal wall, especially in the absence of its edematous changes (dark on dark). Positive contrasts, on the contrary, have an increased signal at T1-WI and T2-WI. This allows you to perfectly visualize the intestinal wall (dark on white), but makes it difficult to analyze contrast images (white on white). Biphasic contrast, having a low signal at T1-WI and a high signal at T2-WI, is devoid of the previously described disadvantages. An example of biphasic contrast is water, but due to the rapid absorption, it does not give good fullness of the intestinal lumen and is therefore not used. The most widely used biphasic contrast, which we use, is a $2.0-2.5 \%$ solution of $₫$ Mannitol, which gives a long and adequate fullness of the intestine for the duration of the scan. In order to qualitatively visualize liquid content, in addition to using the
T1-WI and T2-WI methods, additional signal sequences are used. To assess the state of the intestine, we use the pulse sequence FIESTA (Cor, Ax, Sag) - a coherent technique that uses a full balanced gradient signal (Figure 1). The image contrast is ensured by a high $\mathrm{T} 1 / \mathrm{T} 2$ ratio. That is, substances or tissues that have the maximum difference in signal intensity at T1-WI and T2-WI will be most contrasted with this technique. Accordingly, peroral biphasic contrast enhancement will provide excellent visualization of the intestinal lumen. Images are more T2-weighted. The high scanning speed and very low sensitivity to movement when acquiring images make this technique reliable in patients who have difficulty holding their breath.

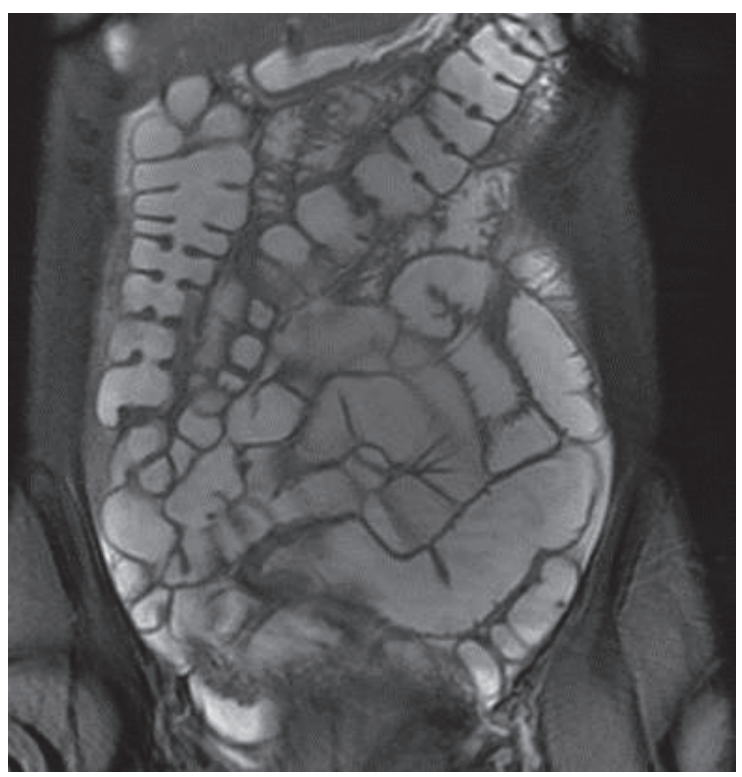

Fig. 1. MR-image of the intestine by FIESTA

Direct methods for determining gastrointestinal activity are electro-gastrography, manometry and the use of electrodes and calibration voltage transducers. The invasiveness of these methods does not allow their frequent use for diagnostic purposes. Conducting dynamic FIESTA provides a unique opportunity to visualize intestinal motility not just in vivo, but also in real time, to evaluate the movement of fluid content in the intestine, to analyze the activity of the ileocecal valve (complete or incomplete closure of its valve flaps, the presence of retrograde current at the level of the small inteFstine transition), slowing down and difficulty in moving the liquid contents in the area of narrowing of the lumen to assess the degree of compensation of stenosis. There is no unified approach to the interpretation of functional studies of the intestine, and the analysis is only quality, but even at this stage of the development of functional techniques, the information received has high diagnostic value. 
Since MRCP (HASTE) is based on the turbo-spin echo technique, which is used to sequentially obtain high-resolution T2-WI and allows to obtain a 3D model of the intestine (Figure 2 ). This allows you to easily select the exact position of the plane to quickly obtain the desired viewing angle and position in space. HASTE uses the technique of short single signals to obtain enough data for the entire image in one sequence repetition period (TR). HASTE imaging uses half-Fourier in phase technology. Only half of the K-space is collected and the remainder is displayed. This technique is essentially an image of exclusively liquid content in the study area - intestinal lumen filled with peroral contrast. Due to the possibility of building a $3 \mathrm{D}$-model, this technique is primarily used to analyze the anatomical pathway of the intestine, verify congenital anomalies, dystopia, dolichocolon and dolichosigma, but also to determine the places of narrowing and expansion of the intestinal lumen. 3D tomography is a modern diagnostic standard, which allowed the quality of diagnosis and treatment to reach a whole new level. The advantage of threedimensional images is the ability to identify defects that are often overlooked in conventional images.

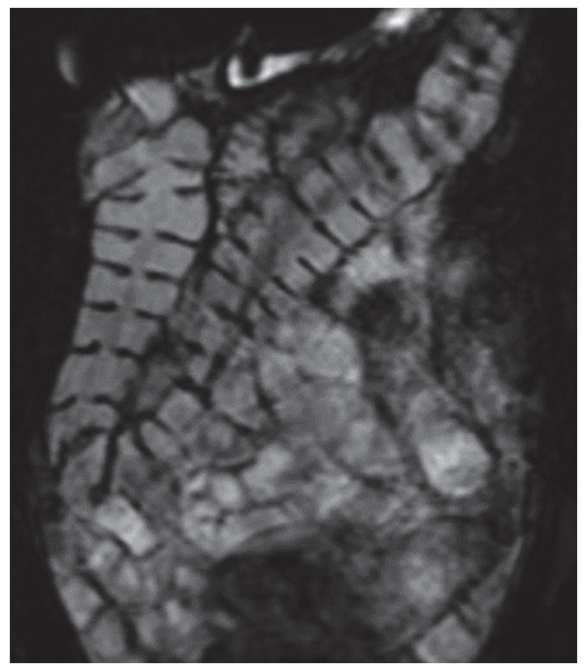

Fig. 2. MR-image of the intestine by MRCP

\section{DISCUSSION}

To obtain MR images of the intestine suitable for analysis and interpretation, it is necessary to fill it with special oral liquid contrasts. Using this technique allows you to visualize not only the small, but also the large intestine throughout its entire length. But because of the high diagnostic significance of the information received on the condition of the small intestine, which is less accessible for diagnosis, this technique is often called MR enterography, although it is more correctly called hydro-MRI due to the use of peroral hydrocontrast. The presence of liquid content allows you to straighten the intestinal wall, improve visualization of its course, wall thickness, the location of loops and folds. Thus, when conducting MR enterography, it is the visualization of liquid content that makes it possible to conduct a qualitative analysis of the state of the intestine and visualize its internal structure. In addition, modern MR technologies allow real-time dynamic studies of the intestine and hydrocontrasting makes it possible to judge the presence of functional changes by changing the rate of advancement of the liquid contents. Based on the data of intestinal MRI, it is possible to create a personalized visualization model of the gastrointestinal tract

\section{REFERENCES}

[1] Westerland O, Griffin N. Magnetic Resonance Enterography in Crohn 's Disease. Semin

Ultrasound CT MR 2016; 37(4):282-2915.

[2] Schukina O.B., Sobko V.Yu. Fecal calprotectin and hydro-MRI in assessing Crohn's disease activity. Bulletin of the North-West State Medical University. I.I. Mechnikov 2013; 5 (1): 78-83.

[3] Trofimova T.N., Dementieva T.V., Karpenko A.K. Possibilities of modern methods of radiation diagnostics in visualization of the colon in the norm and with its pathological changes: CT-colonography // Radiation diagnostics and therapy.-2010.- No. 2 .- S. 64-68.

[4] Draft clinical guidelines for the diagnosis and treatment of inflammatory bowel disease. Coloproctology 2013; 3 (45): 40 